\title{
Altered expression of differential gene and IncRNA in the lower thoracic spinal cord on different time courses of experimental obstructive jaundice model accompanied with altered peripheral nociception in rats
}

\author{
Qian Wang ${ }^{1, *}$, Zhi-Xiao Li ${ }^{1,}{ }^{*}$, Bao-Wen Liu ${ }^{1,}{ }^{*}$, Zhi-Gang He ${ }^{1}$, Cheng Liu ${ }^{1}$, Min Chen ${ }^{2}$, \\ San-Guang Liu ${ }^{3}$, Wei-Zhong $\mathbf{W u}^{4}$ and Hong-Bing Xiang ${ }^{1}$ \\ ${ }^{1}$ Department of Anesthesiology and Pain Medicine, Tongji Hospital, Tongji Medical College, Huazhong University of Science \\ and Technology, Wuhan, P.R. China \\ ${ }^{2}$ Department of Anesthesiology, Hubei Maternal and Child Health Hospital, Wuhan, P.R. China \\ ${ }^{3}$ Department of Hepatobiliary Surgery, The Second Hospital, Hebei Medical University, Shijiazhuang, P.R. China \\ ${ }^{4}$ Department of General Surgery, The Second Hospital, Hebei Medical University, Shijiazhuang, P.R. China \\ * These authors have contributed equally to this work and should be considered co-first authors \\ Correspondence to: Wei-Zhong Wu, email: wwzdhr@sohu.com \\ Keywords: obstructive jaundice; nociceptive response; high-throughput sequencing; IncRNA; spinal cord \\ Received: August 29, $2017 \quad$ Accepted: October 28, $2017 \quad$ Published: November 20, 2017 \\ Copyright: Wang et al. This is an open-access article distributed under the terms of the Creative Commons Attribution License 3.0 \\ (CC BY 3.0), which permits unrestricted use, distribution, and reproduction in any medium, provided the original author and source \\ are credited.
}

\section{ABSTRACT}

The spinal origin of jaundice-induced altered peripheral nociceptive response poorly understood. In the current study, we aimed to first validate rats with bile duct ligation (BDL) as a jaundice model accompanied by altered peripheral nociceptive response, and then to analyze differential gene and IncRNA expression patterns in the lower thoracic spinal cord on different time courses after BDL operation by using high-throughput RNA sequencing. The differentially expressed genes (DEGs) identified using reverse transcription-quantitative polymerase chain reaction (RT-qPCR) analysis, followed by clustering analysis, Gene Ontology analysis and pathway analysis. As a result, a total of 2033 IncRNAs were differentially expressed 28d after BDL, in which 1545 probe sets were up-regulated and 488 probe sets were down-regulated, whereas a total of $\mathbf{2 8 0 0}$ mRNAs were differentially expressed, in which 1548 probe sets were upregulated and 1252 probe sets were down-regulated. The RNAseq data of select mRNAs and IncRNAs was validated by RT-qPCR. 28d after BDL, the expressions of IncRNA NONRATT002335 and NONRATT018085 were significantly up-regulated whereas the expression of IncRNA NONRATT025415, NONRATT025388 and NONRATT025409 was significantly down-regulated. 14d after BDL, the expressions of IncRNA NONRATT002335 and NONRATT018085 were significantly up-regulated; the expression of IncRNA NONRATT025415, NONRATT025388 and NONRATT025409 was significantly downregulated. In conclusion, the present study showed that jaundice accompanied with decreased peripheral nociception involved in the changes of gene and IncRNA expression profiles in spinal cord. These findings extend current understanding of spinal mechanism for obstructive jaundice accompanied by decreased peripheral nociception.

\section{INTRODUCTION}

Obstructive jaundice is a clinical syndrome related to bile cholestasis and is governed by complex signals
[1-3]. While historically studied by researching the obstructive jaundice, inputs from the "jaundiced live" and cholestasis impact many functional conditions [4-6]. Indeed, emerging data suggest that significant progress has 
been made in understanding the communication between the cholestatic liver diseases and spinal cord in jaundice regulation [7-10]. The spinal cord is well known to be a complex community of specific genes and long noncoding RNAs (lncRNAs) [11-13] that profoundly influence many aspects of occurrence and development for pathologic disorders [14-20], including development of the jaundice regulation. Though there are various studies in clinical disorders by obstructive jaundice, its differential gene and lncRNA expression pattern in the thoracic spinal cord remains unknown.

The introduction of high-throughput RNA sequencing (RNA-seq), where thousands of differential genes and lncRNA expression patterns can be studied in parallel [21-24], permits a broad assessment for altered expression of differential gene and lncRNA in the lower thoracic spinal cord on different time courses of obstructive jaundice model. In current study, we performed a comprehensive transcriptome analysis in obstructive jaundice model using RNA-seq, and identified lncRNAs with differential expression. To explore the function of lncRNAs, we predicted their potential targets with cis-regulatory effects, which were then put into gene ontology (GO) and Kyoto Encyclopedia of Genes and Genomes (KEGG) for further analysis. In addition, we compared the expression of some lncRNAs in thoracic spinal cord at different time points in obstructive jaundice model.
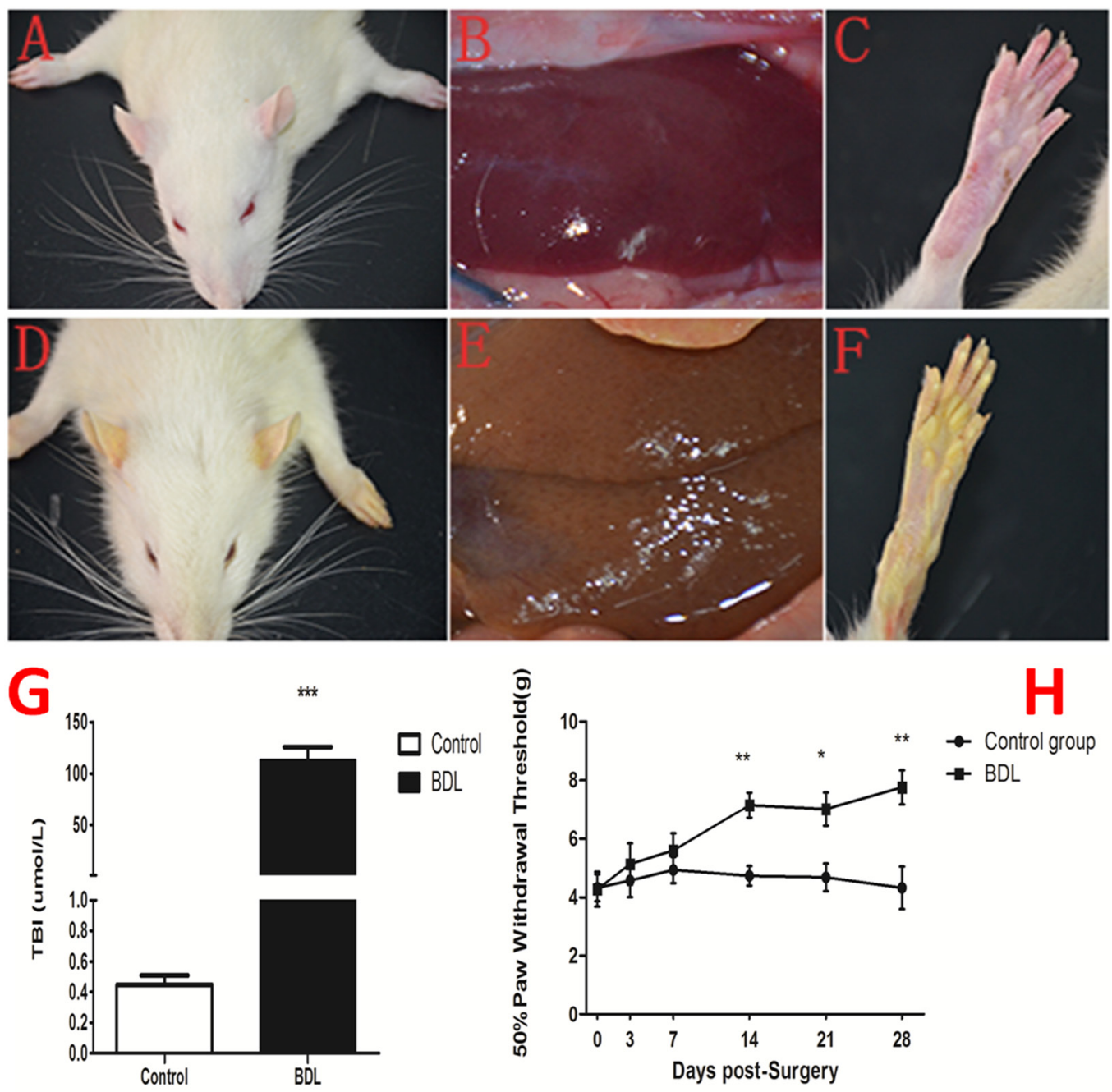

Figure 1: Evaluation of jaundice and the change of nociceptive threshold following BDL operation. (A-F) The rats in BDL group showed yellow ears (D), liver tissue(E) and rear claw(F) 28 days after surgery compared to control group (A, B, C). (G) The serum total bilirubin levels was significantly higher in the BDL group $(112.8 \pm 12.88 \mu \mathrm{ml} / \mathrm{L})$ than in the control group $(0.45 \pm 0.06 \mu \mathrm{ml} / \mathrm{L})$. $(\mathbf{H}) \mathrm{The}$ mechanical PWT was tested to assess the jaundice-induced nociceptive threshold change prior to (day 0 ) and at days $3,7,14,21$, and 28 following BDL operation. The hindpaw of BDL rats displayed a dramatic decrease in mechanical PWT to von Frey filament stimulation from $4.28 \pm 0.59 \mathrm{~g}$ at baseline to $7.15 \pm 0.43 \mathrm{~g} 14 \mathrm{~d}$ after BDL operation and further deteriorated to $7.02 \pm 0.57$ at day 21 , and $7.76 \pm 0.58 \mathrm{~g}$ at day 28. ${ }^{*} \mathrm{P}<0.05,{ }^{* *} \mathrm{P}<0.01,{ }^{* * *} \mathrm{P}<0.001$ compared with the each corresponding time point. 
A

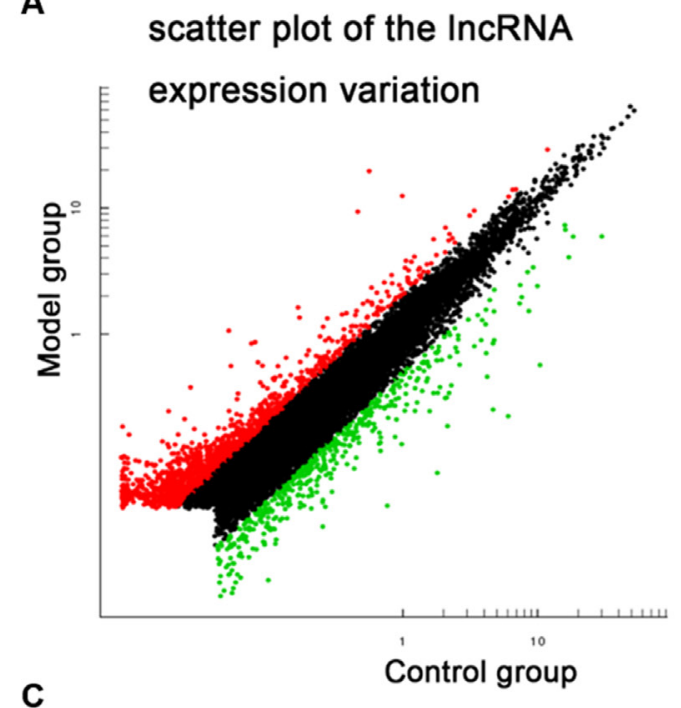

C<smiles>C1=C[C@H]2C=C[C@H]1C2</smiles>

IncRNA

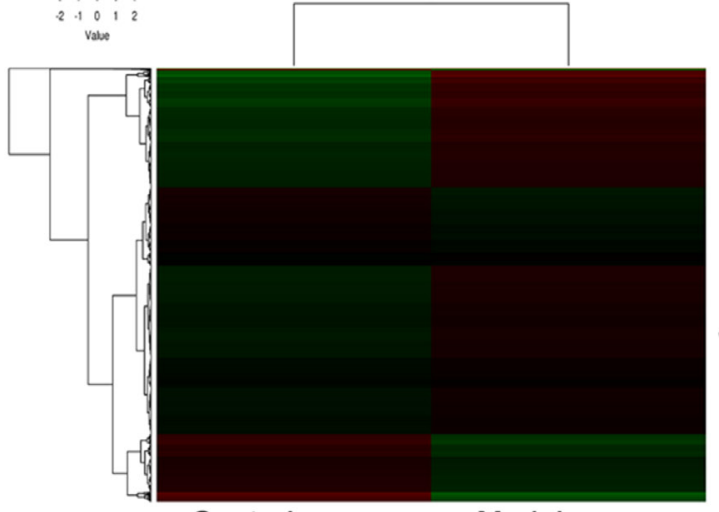

Control group Model group

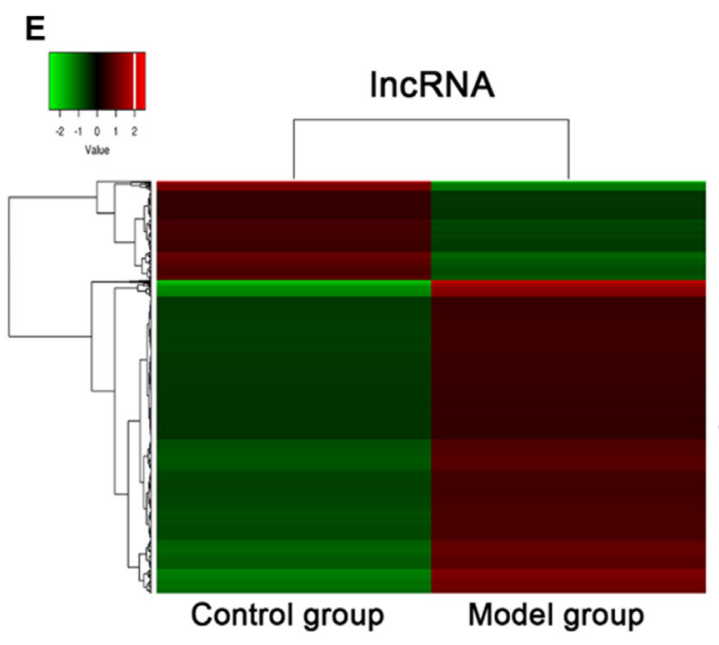

B
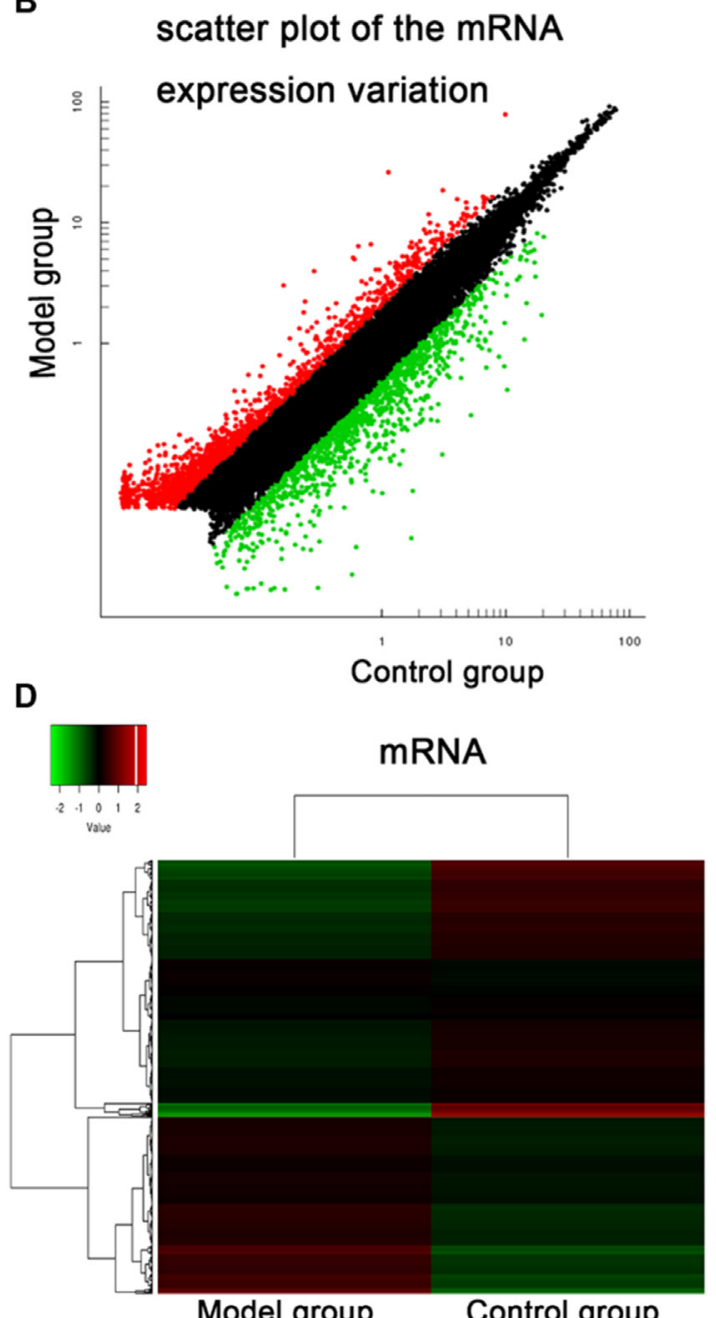

$\mathbf{F}$

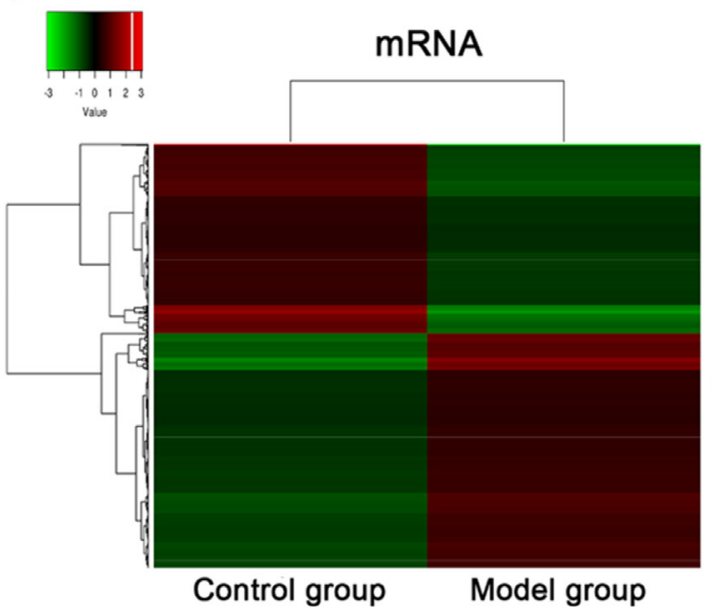

Figure 2: Common bile duct ligation resulted in the expression profiling changes of IncRNA and mRNA. Scatter plot comparing global mRNA (A) gene expression profiles in the spinal cord between BDL group (Model group) and sham group (Control group) rats; Red color is indicative of up-regulated and green color is indicative of down-regulated genes. Black color is indicative of not statistical significant different genes when it does not pass the cutoff values of 1 and -1 in $\log 2$ scale. Heat map showing hierarchical clustering of overall mRNA (B) expression pattern of reliably measured probe sets. Heat map showing hierarchical clustering of mRNA (C), whose expression changes were more than twofold. In clustering analysis, up- and down-regulated genes are colored in red and green, respectively. 
Table 1: The detail information of the top 20 up-regulated and 20 down-regulated lncRNAs

\begin{tabular}{lccc}
\hline $\begin{array}{l}\text { Up-regulated } \\
\text { IncRNAs }\end{array}$ & $\begin{array}{c}\text { Log fold change } \\
\text { (Model/control) }\end{array}$ & $\begin{array}{c}\text { Down-regulated } \\
\text { IncRNAs }\end{array}$ & $\begin{array}{c}\text { Log fold change } \\
\text { (Model/control) }\end{array}$ \\
\hline NONRATT025327 & 35.01176765 & NONRATT025415 & -27.18649832 \\
NONRATT000845 & 22.26444654 & NONRATT025388 & -22.79828 \\
NONRATT002335 & 20.87539552 & NONRATT025409 & -18.60605684 \\
NONRATT018085 & 20.23490953 & NONRATT025389 & -18.42176407 \\
gi|672030539|ref|XR_598272.1| & 17.2076069 & NONRATT006517 & -17.6681701 \\
NONRATT001654 & 14.26208272 & NONRATT012834 & -11.53074647 \\
NONRATT006896 & 13.42852291 & NONRATT028806 & -9.221338198 \\
gi|672021044|ref|XR_347474.2| & 12.94719615 & gi|672015760|ref|XR_599773.1| & -9.002940997 \\
NONRATT022152 & 12.644969 & gi|672089057|ref|XR_597824.1| & -8.581978626 \\
NONRATT030272 & 12.62142301 & NONRATT018084 & -7.331131967 \\
gi|672019340|ref|XR_601027.1| & 12.55900993 & NONRATT023718 & -6.747783528 \\
gi|672029209|ref|XR_596791.1| & 11.512809 & NONRATT031185 & -6.642754333 \\
NONRATT011339 & 11.21739711 & NONRATT029420 & -6.473944169 \\
NONRATT027405 & 11.11711302 & NONRATT010390 & -6.470431266 \\
NONRATT028521 & 10.78646213 & NONRATT020020 & -6.22851892 \\
NONRATT006730 & 10.55666403 & NONRATT013131 & -6.121677607 \\
gi|672016774|ref|XR_600156.1| & 10.39316965 & NONRATT006732 & -5.906932924 \\
gi|672079892|ref|XR_596396.1| & 10.08651117 & NONRATT019209 & -5.848382084 \\
gi|672024744|ref|XR_599302.1| & 10.0551937 & NONRATT022428 & -5.771813271 \\
gi|672033238|ref|XR_349371.2| & 9.84667827 & NONRATT022429 & -5.730278642 \\
\hline
\end{tabular}

\section{RESULTS}

\section{Evaluation of jaundice}

We observed the general appearance of the animals used in this study (Figure 1), and found that rats in BDL group showed yellow ears (D), liver tissue(E) and rear claw(F) 28 days after surgery compared to control group $(\mathrm{A}, \mathrm{B}, \mathrm{C})$. Otherwise, our results showed that the serum total bilirubin levels was significantly higher in the BDL group $(112.8 \pm 12.88 \mu \mathrm{ml} / \mathrm{L})$ than in the control group $(0.45 \pm 0.06 \mu \mathrm{ml} / \mathrm{L})$ (Figure $1 \mathrm{G})$.

\section{The change of nociceptive threshold following BDL operation}

The mechanical nociceptive stimulation was tested to assess the jaundice-induced nociceptive threshold change prior to (day 0 ) and at days $3,7,14,21$, and 28 following BDL operation. Our result showed that the hindpaw of BDL rats displayed a dramatic increase in mechanical nociceptive threshold to von Frey filament stimulation from $4.28 \pm 0.59 \mathrm{~g}$ at baseline to $7.15 \pm 0.43 \mathrm{~g}$ $14 \mathrm{~d}$ after BDL operation and further deteriorated to 7.02 \pm 0.57 at $21 \mathrm{~d}$ and $7.76 \pm 0.58 \mathrm{~g}$ at $28 \mathrm{~d}$ after BDL operation
(Figure 1H). These data indicated that obstructive jaundice resulted in the decreased sensitivity in response to the mechanical nociceptive stimulation in the present study.

\section{Differentially expressed genes of T6-T12 spinal cord 28d after BDL operation}

To systematically identify jaundice-associated lncRNAs in spinal cord, the differential expression analysis was performed between BDL group and control group. The lncRNA and mRNA expressions of T6-T12 spinal cord of the animals were examined the HiSeq 2000 system (Illumina, Inc.) with a total of 1,198,903,526 raw reads from the nine libraries. Differentially expressed gene profiles in BDL group were compared to the corresponding data from control group. Figure 2 indicated the scatter plot comparing global mRNA (A) gene expression profiles in the spinal cord between BDL group (Model group) and sham group (Control group), and heat map showing hierarchical clustering of overall mRNA (B) expression pattern of reliably measured probe sets. In addition, heat map also showed hierarchical clustering of mRNA (C), whose expression changes were more than twofold. 
Table 2: The detail information of the top 20 up-regulated and 20 down-regulated mRNAs

\section{Gene symbol \\ Up-regulated genes}

Description $\log _{2}$ fold change

(Model/control)
Gemin8

Serpina $3 n$

Trim63

Olr728

Hif3a

Olr666

Olr1206

Retnla

Ilost

Retnlb

Cxcl17

$\operatorname{Reg} 3 b$

Olr383

Olr1192

Olr1075

Tas2r114

Zbtb16

RGD1309489

Olr1063

Olr215

\section{Down-regulated genes}

Shisa3

Sostdc1

Sfrp 4

Smoc2

Smoc2

Mfap4

Colla1

Gxylt2

Coll5al

Igfbp5

Selp

Cdh1

Coll2al

Scin gem (nuclear organelle) associated protein 8

serine (or cysteine) peptidase inhibitor, clade A, member $3 \mathrm{~N}$

tripartite motif containing 63, E3 ubiquitin protein ligase

olfactory receptor 728

hypoxia inducible factor 3 , alpha subunit

olfactory receptor 666

olfactory receptor 1206

resistin like alpha

interleukin 6 signal transducer

resistin like beta

chemokine (C-X-C motif) ligand 17

regenerating islet-derived 3 beta

olfactory receptor 383

olfactory receptor 1192

olfactory receptor 1075

taste receptor, type 2, member 114

zinc finger and BTB domain containing 16

similar to Est1p-like protein B

olfactory receptor 1063

olfactory receptor 215

shisa family member 3

sclerostin domain containing 1

secreted frizzled-related protein 4

SPARC related modular calcium binding 2

SPARC related modular calcium binding 2

microfibrillar-associated protein 4

collagen, type I, alpha 1

glucoside xylosyltransferase 2

collagen, type XV, alpha 1

insulin-like growth factor binding protein 5

selectin $\mathrm{P}$

cadherin 1

collagen, type XII, alpha 1

scinderin
23.14275154

13.96190308

10.67545524

10.23434831

9.861685919

9.644311752

9.628587408

9.376262216

9.164598351

9.109799277

8.725497102

8.598931015

8.574541671

8.391854793

8.369125332

8.360873477

8.294338675

8.266135149

8.240355839

8.230039718

$-71.14729446$

$-47.37907925$

$-32.38831334$

$-29.71653174$

$-25.62228109$

$-25.00123131$

$-20.63943781$

$-18.03100637$

$-17.56597588$

$-15.10090131$

$-14.44927067$

$-14.28981311$

$-14.02389161$

$-14.00885112$

(Continued) 


\begin{tabular}{lcc}
\hline Gene symbol & Description & $\begin{array}{c}\mathbf{L o g}_{2} \text { fold change } \\
\text { (Model/control) }\end{array}$ \\
\hline Wisp1 & WNT1 inducible signaling pathway protein 1 & -13.78032056 \\
Col6a3 & collagen, type VI, alpha 3 & -13.72869989 \\
Col3a1 & collagen, type III, alpha 1 & -13.16577469 \\
Loxl1 & lysyl oxidase-like 1 & -12.37937746 \\
Smim5 & small integral membrane protein 5 & -11.67644662 \\
Prdm6 & PR domain containing 6 & -11.448275 \\
\hline
\end{tabular}

A

B
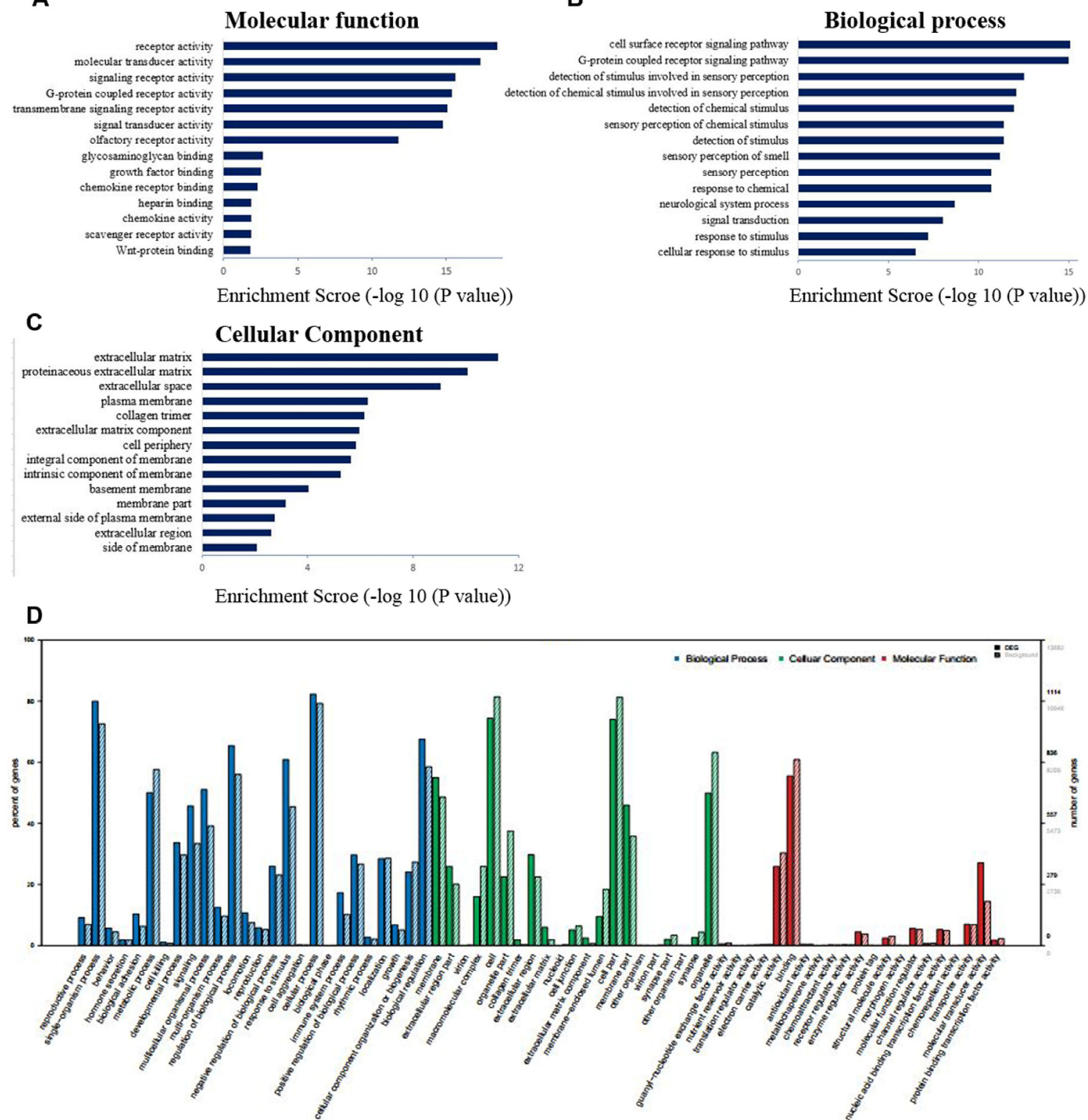

Figure 3: The differential expression of mRNAs in spinal cord after common bile duct ligation-induced jaundice model was analyzed by Gene Ontology (GO) annotation and enrichment. (A) molecular function classification; (B) biological process classification; (C) cellular component classification. (D) The differential expression genes were analyzed with GO background significant enrichment. Solid bar represents target genes set, slash bar represents genes set. 
We identified that a total of $2033 \operatorname{lncRNAs}$ were differentially expressed between control group and BDL group, in which 1545 probe sets were up-regulated and 488 probe sets were down-regulated, whereas a total of 2800 mRNAs were differentially expressed between control group and BDL group, in which 1548 probe sets were upregulated and 1252 probe sets were down-regulated. The detail information of the top 20 up-regulated and 20 downregulated lncRNAs was in Table 1 , and that of the top 20 up-regulated and 20 down-regulated mRNAs was in Table 2. The maximal and minimal fold change was 35.01 and 2.01 , respectively.

\section{Gene ontology annotation for differential expression genes}

We had used the RNA-seq analyses to identify the differentially expressed genes which were annotated using the GO database (Gene Ontology, http://www. geneontology.org/). The present study showed three important results involved in biological functional groups including molecular function (Figure 3A), biological process (Figure 3B), and cellular component (Figure $3 \mathrm{C})$. Figure 3D indicated that the differential expression genes were analyzed with GO background significant enrichment, and the differentially expressed mRNAs in spinal cord were primarily involved in the biological processes GO functions (Figure 4).

\section{Validation of the IncRNAs by RT-qPCR 28d} after BDL operation

To validate the RNA-seq data, top 5 up-regulated lncRNAs (NONRATT025327, NONRATT000845, NONRATT001654, NONRATT002335, and NONRATT018085) and top 5 down-regulated lncRNAs (NONRATT025415, NONRATT025388, NONRATT025409, NONRATT02538 and NONRATT006517) that were differentially expressed between BDL group and control group were randomly selected (Table 3), and their relative expression levels were quantified by RT-qPCR. We found that the expressions of IncRNA NONRATT002335 and NONRATT018085 were significantly up-regulated underlying BDL group compared with control group, whereas the expression of IncRNA NONRATT025415, NONRATT025388 and NONRATT025409 was significantly down-regulated in model group (Figure 5).

\section{The expression of 10 IncRNAs in T6-T12 spinal cord at different time points (14d/28d) after BDL operation}

Obviously, gene expressions after BDL operation injection are varied in different time points. We collected spinal tissue sample from T6-T12 spinal cord $14 \mathrm{~d}$ vs $28 \mathrm{~d}$ after BDL operation for RT-qPCR

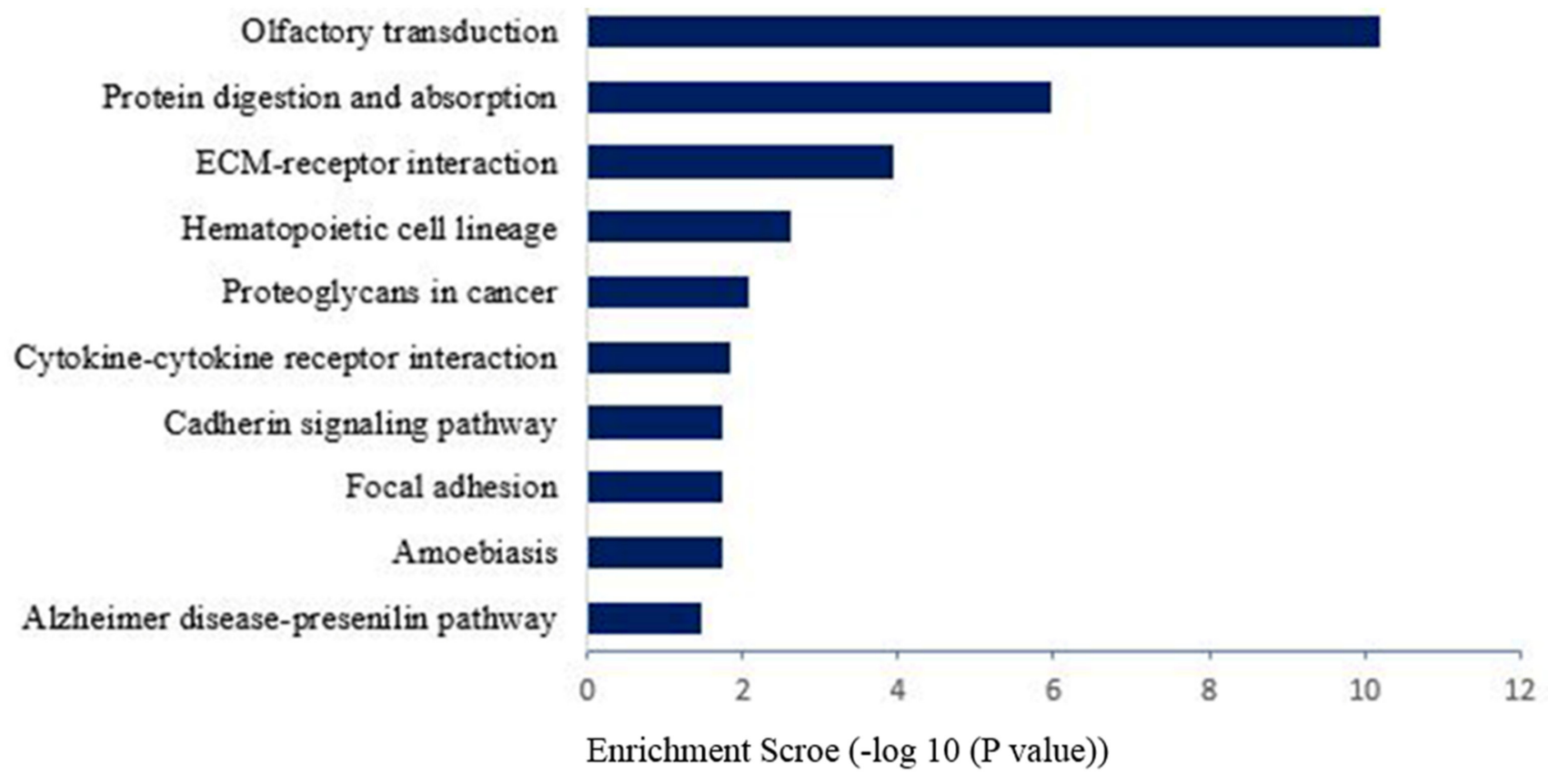

Figure 4: Pathway analysis for differential expression of mRNAs in spinal cord after common bile duct ligationinduced jaundice model. 
Table 3: Top 5 up-regulated IncRNAs

\begin{tabular}{lccc}
\hline ProbeName & $\begin{array}{c}\text { log2Fold change (Model group/ } \\
\text { control group) }\end{array}$ & GeneID & Product length \\
\hline NONRATT025327 & 35.0117676468596 & NONRATG020647.2 & 150 \\
NONRATT000845 & 22.2644465403801 & NONRATG000689.2 & 212 \\
NONRATT002335 & 20.8753955158768 & NONRATG001912.2 & 150 \\
NONRATT018085 & 20.2349095300728 & NONRATG014750.2 & 81 \\
NONRATT001654 & 14.2620827239878 & NONRATG001356.2 & 132 \\
Top 5 down-regulated lncRNAs & & & 120 \\
NONRATT025415 & 27.1864983217999 & NONRATG020725.2 & 89 \\
NONRATT025388 & 22.7982799999892 & NONRATG020701.2 & 111 \\
NONRATT025409 & 18.6060568387294 & NONRATG020719.1 & 131 \\
NONRATT025389 & 18.4217640674687 & NONRATG020702.2 & 95 \\
NONRATT006517 & 17.6681700975811 & NONRATG005240.2 & \\
\hline
\end{tabular}
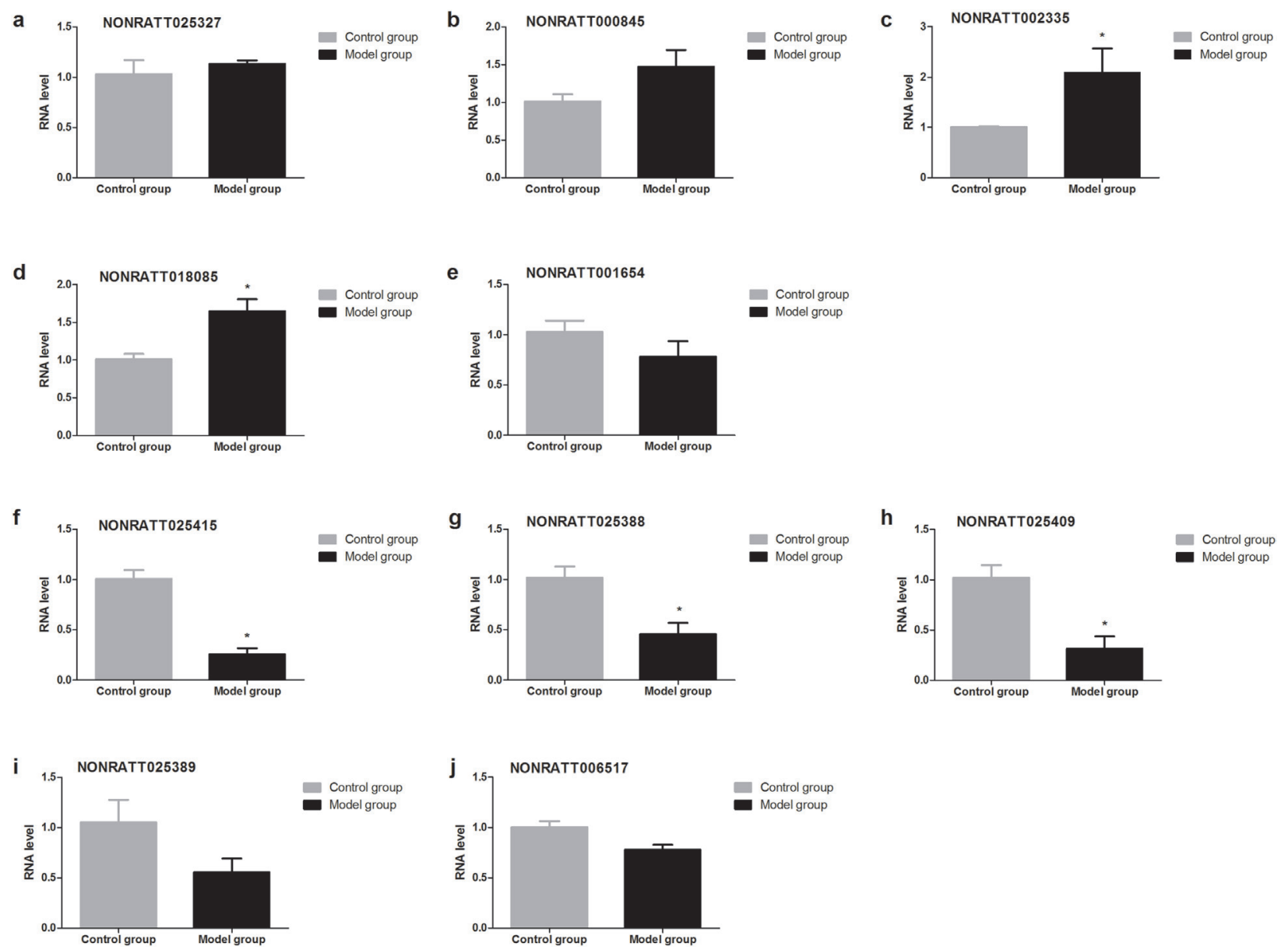

Figure 5: Validation of the IncRNAs by RT-qPCR 28d after BDL operation. The expressions of lncRNA NONRATT002335 and NONRATT018085 were significantly up-regulated underlying BDL group compared with control group, whereas the expression of IncRNA NONRATT025415, NONRATT025388 and NONRATT025409 was significantly down-regulated in model group. 
validation. 14d after BDL operation, the expressions of IncRNA NONRATT002335 and NONRATT018085 were significantly up-regulated underlying BDL group compared with control group whereas the expression of IncRNA NONRATT025327, NONRATT000845 and NONRATT001654 had not statistically different between control group and BDL group; The expression of IncRNA NONRATT025415, NONRATT025388 and NONRATT025409 was significantly down-regulated underlying BDL group compared with control group whereas the expression of lncRNA NONRATT02538 and NONRATT006517 had not statistically different between control group and BDL group (Figures 6 and 7).

\section{DISCUSSION}

In the present study, we confirmed that bile duct ligation resulted in obstructive cholestasis and jaundice and this model was consistent with previous reports describing marked elevation of the serum total bilirubin
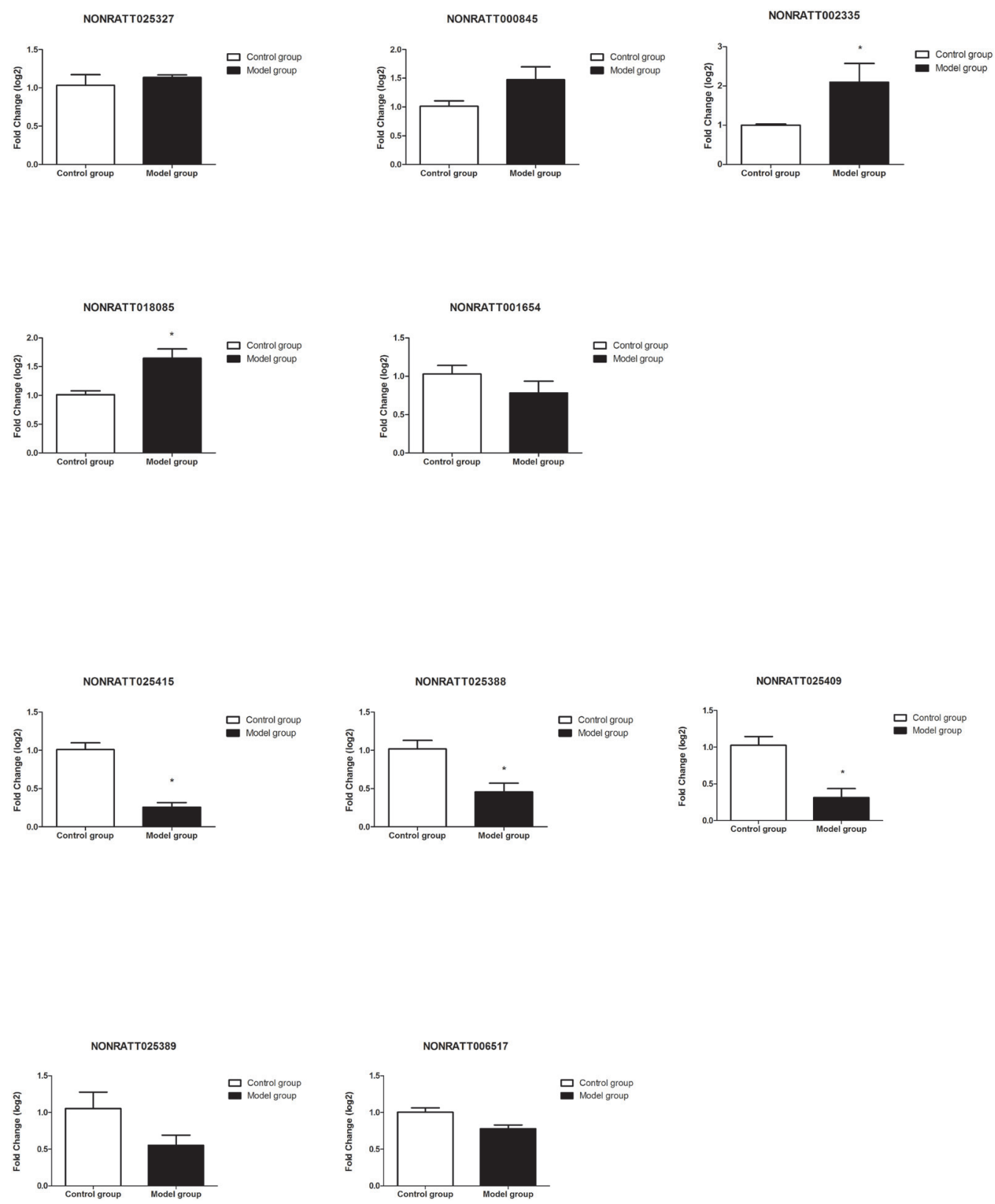

Figure 6: Validation of the IncRNAs by RT-qPCR 14d after BDL operation. The expressions of lncRNA NONRATT002335 and NONRATT018085 were significantly up-regulated underlying BDL group compared with control group; the expression of lncRNA NONRATT025415, NONRATT025388 and NONRATT025409 was significantly down-regulated underlying BDL group compared with control group whereas the expression of lncRNA NONRATT02538 and NONRATT006517 had not statistically different between control group and BDL group. 
in rats responding to bile duct ligation $[5,25]$. In addition, our result also indicated that obstructive jaundice induced the decreased sensitivity in response to the mechanical nociceptive stimulation $14 \mathrm{~d}$ and $28 \mathrm{~d}$ after BDL operation, which was in line with a previous experimental study showing that obstructive cholestasis by bile duct resection in rodents displayed decreased nociception [26-28].
We identified the spinal genes and lncRNAs that were differentially expressed between model group and control group by high throughput RNA-seq, providing an important view of spinal genetic heterogeneity for jaundice-induced decreased nociception in rats. The present study identified 2033 differentially expressed lncRNAs in the spinal cord, 488 of which were down-
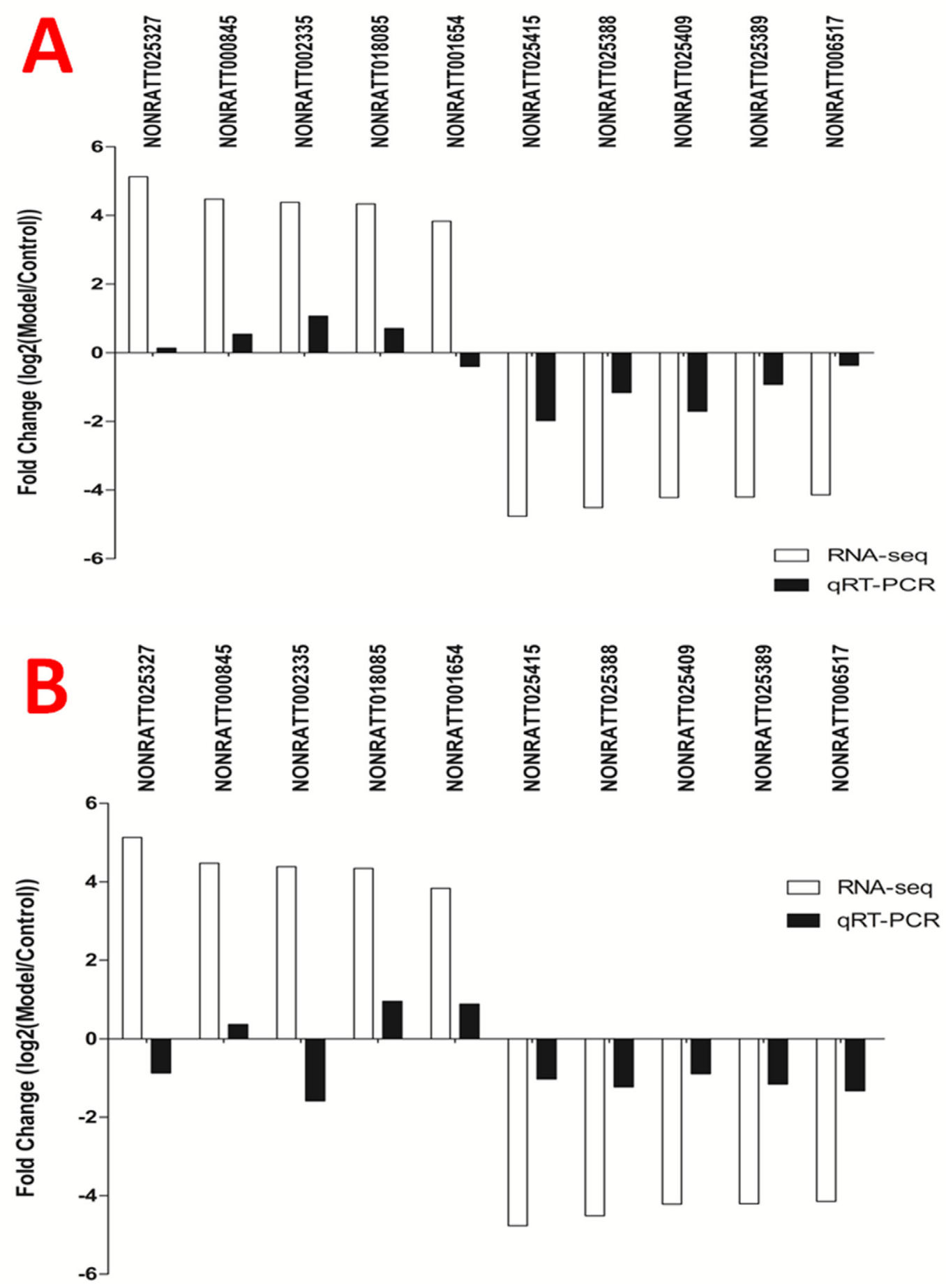

Figure 7: The expression of 10 IncRNAs in T6-T12 spinal cord at different time points after BDL operation. (A) 14d after BDL operation, four up-regulated lncRNAs and five down-regulated lncRNAs were validated by RT-qPCR. (B) 28 d after BDL operation, three up-regulated lncRNAs and five down-regulated lncRNAs were validated by RT-qPCR. 
Table 4: Primer sequences for RT-qPCR

\begin{tabular}{|c|c|c|}
\hline Gene & Forward (5' to $\left.3^{\prime}\right)$ & Reversed (5' to $\left.3^{\prime}\right)$ \\
\hline NONRATT025327 & ATGCACCGCTAGAAGTCACAGA & GCTCAGACCCAGTTGCTCCC \\
\hline NONRATT000845 & AGTAAACCCCTAACAAATCCCC & GGTCACCTAAGACCATTGGAAA \\
\hline NONRATT002335 & AGCCATGCGTCTTCCTAACC & AGCACCTGAACAAGCCACCT \\
\hline NONRATT018085 & ACCAGATGGAACGATTAAACCC & CCACTGCCACTGAACCTTGA \\
\hline NONRATT001654 & GGGTGGCTGGATTTCATTTT & GGGGAACAGAGGGAATACAACA \\
\hline NONRATT025415 & CTGGTCTGGGTTTTCCTTCTTG & TGAGGTGGCACAGGTGAGTTT \\
\hline NONRATT025388 & TCCACTGATTTCCAGGCTCTT & GCCCTTCACTTGACACCTACAC \\
\hline NONRATT025409 & AGGGCTTGAAGTGGATGGG & TTGCAGTGCTGGCAGAGG \\
\hline NONRATT025389 & ATTGACTCTGGCCTGGGAGC & TTATGGTGCAAGTGAGGTTGG \\
\hline NONRATT006517 & ATCCAAAATGATTTCCTACCCA & GACCAGCTATAAGCCAGTGTCC \\
\hline GAPDH & CGCTAACATCAAATGGGGTG & TTGCTGACAATCTTGAGGGAG \\
\hline
\end{tabular}

RT-qPCR: reverse transcriptase quantitative polymerase chain reaction

regulated $>2$-fold whereas 1545 of which were upregulated $>2$-fold. Similarly, a total of 2800 mRNAs were differentially expressed between control group and BDL group, 1252 of which were down-regulated whereas 1548 of which were up-regulated $(\mathrm{FDR}<0.05,|\log 2 \mathrm{FC}|>1)$. Among top 5 up-regulated lncRNAs and top 5 downregulated lncRNAs, 2 up-regulated (NONRATT002335 and NONRATT018085) and 3 down-regulated lncRNAs (NONRATT025415, NONRATT025388 and NONRATT025409) were identified. These results suggested that $50 \%$ differentially expressed lncRNAs were verified by RT-qPCR. Furthermore, we found that Serpina3n was an important up-regulated gene $28 \mathrm{~d}$ after BDL operation, which were in agreement with a previous study that the serine protease inhibitor Serpina3n was upregulated in the dorsal root ganglia (DRG) after nerve injury, and attenuated neuropathic pain by inhibiting $\mathrm{T}$ cell-derived leukocyte elastase [29].

Previous studies have suggested that the different duration of cholestasis accompanies by some behavioral change in rodents $[26,30]$. Belghiti et al observed that chronic BDL rats displayed enhanced scratching behavior and thermal hyperalgesia already $48 \mathrm{~h}$ after BDL surgery, and this result was perfectly demonstrated by the upregulation and sensitization of the heat-sensitive TRPV1 channel [30]. Tian et al also demonstrated 5-HT-induced enhanced scratching and antinociception to mechanical and heat stimuli in BDL rats [31], suggesting that targeting 5-HT receptors may be an effective treatment for cholestatic itch. In this study, we found differential expression of some lncRNAs (Figure 7) in the spinal cord at different time points $(14 \mathrm{~d} / 28 \mathrm{~d})$ after BDL operation, suggesting that the different lncRNAs in spinal cord segment may be involved in the neuronal response to obstructive jaundice. Although the functions of many
lncRNAs in spinal cord are not fully known, our findings provide novel potential insights involving in the molecular mechanism of jaundice-induced altered peripheral nociception.

In conclusion, obstructive jaundice accompanied by altered peripheral nociception is always a crucial factor limiting the therapeutic efficacy of many drugs and quality of life for patients. Here we constructed the expression profiles of lncRNAs and potentially related mRNAs in rats with obstructive jaundice, and found some distinct lncRNA/mRNA expression profiles in spinal cord, suggesting that these unique noncoding transcripts may contribute to the acquisition of cholestasis-induced altered peripheral nociception. Although additional studies are needed to verify these lncRNAs/mRNAs mentioned above, our study provides important insights into novel indicators of treatment for patients with cholestasis.

\section{MATERIALS AND METHODS}

\section{Animal care}

Male Sprague-Dawley rats were provided by the Center of Experimental Animal of Tongji Medical College (license number: 43004700019962). All experimental protocols, animal testing and surgeries were performed in adherence to the National Institute of Health Guide for the Care and Use of Laboratory Animals (NIH Publications No. 80-23) revised 1996. The experimental protocols were approved by the committee of experimental animals of Tongji Hospital, Tongji Medical College (IRBID:TJ-A0804). The animals were maintained in a climate controlled room on a 12-hlight/dark cycle (light on at 07:00 h). Rats were housed (2/cage), but they were 
individually caged during each experiment. Food and water were available ad libitum.

\section{Obstructive jaundice model by bile duct ligation}

Rats were placed in a temperature-controlled chamber before experimental operation. All surgical procedures were performed under sterile conditions. Common bile duct ligation (BDL) established the prolonged obstructive jaundice as described in previous studies [32-34]. After an intraperitoneal injection of ketamine hydrochloride $(50 \mathrm{mg} / \mathrm{kg})$ plus xylazine $(5 \mathrm{mg} /$ $\mathrm{kg}$ ), rats which underwent BDL were anesthetized. In those with BDL, the common bile duct was located and ligated using 4-0 silk at two points anterior to the pancreas and posterior to the hilum of the liver. The first ligation was made just above the duodenum and the second almost $2 \mathrm{~mm}$ above the first ligation. The bile duct was then transected at the midpoint between the two ligatures. Animals used in this study were sacrificed before signs of severe illness became apparent.

\section{Experimental groups}

Experiment A Rats were randomly assigned to two groups: (1) Control group (sham-ligation surgery, $n$ =9); (2) BDL group (common bile duct ligation, $\mathrm{n}=9$ ). The mechanical nociceptive thresholds were evaluated at different time points including day (d) 0 (baseline), 3, 7, 14,21 , and 28. 28d after operation, rats were sacrificed, and blood samples, spinal cord tissues were collected for further analysis. Serum total and direct bilirubin levels were measured. Thoracic segments of spinal cord (T6T12) were prepared to analyze differential gene and IncRNA expression patterns by high-throughput RNA sequencing, and thoracic (T6-T12) segment of spinal cord for Real-Time quantitative PCR (RT-qPCR).

Experiment B Rats were randomly assigned to two groups: (1) Control group ( $\mathrm{n}=12)$; (2) BDL group $(\mathrm{n}=12) .14$ days after surgery the T6-T12 spinal cord were collected using a dissection microscope, rinsed with isotonic saline, dissected and fleshly frozen in liquid nitrogen for RT-qPCR.

\section{Assessment of mechanical sensitivity}

As in previous reports [35-38], mechanical paw withdraw threshold was examined using the blind method with a minimum of six animals/group. Calibrated von Frey filament apparatus (Stoelting, Wood Dale, IL, USA) was used to measure the paw withdrawal response for a mechanical stimulus. A range of filament comprises $1 \mathrm{~g}$, $1.4 \mathrm{~g}, 2 \mathrm{~g}, 4 \mathrm{~g}, 6 \mathrm{~g}, 8 \mathrm{~g}, 10 \mathrm{~g}$, and $15 \mathrm{~g}$ bending force. Rats were allowed to acclimate within a clear plastic chamber over an elevated mesh floor for 30 minutes before assessment at room temperature. Each filament was applied to the midplantar surface of the left hind paw until a withdrawal response occurred. To avoid tissue injury in refractory animals, stimulation was automatically terminated after 15 seconds. The interval between adjacent tests was $>5$ minutes. The results of 3 consecutive measurements were averaged for the mechanical paw withdraw threshold in BDL and control rats.

\section{Analysis of jaundice index}

28d following BDL operation, serum total bilirubin levels were determined in the Automated Blood Chemical Analyzer Vitro 350 (Orthoclinical Diagnostic Inc., Rochester, NY).

\section{RNA extraction, library preparation and high- throughput RNA sequencing}

RNA was isolated from the thoracic (T6-T12) spinal segment tissues in two groups using TRI reagent (Invitrogen, Carlsbad, CA, USA) according to the manufacturer's instructions. Tissue samples were determined using a NanoDrop 2000 (Thermo Fisher Scientific Inc, USA) for integrity, quality and purity.

Library preparation and high-throughput RNA sequencing were performed by the OE Biotechnology Corporation (Shanghai, China) [39-41]. Total RNA of all samples were sequenced by EMBL GeneCore (EMBL Genomics Core Facility, Heidelberg, Germany). The synthesis of polyadenylated transcriptome libraries for every sample was accompanied by deep sequencing in 3 lanes each generating $>50$ million reads pair end. RNAseq was performed in the two groups, each with three biological replicates.

\section{Real-time quantitative-PCR}

Thoracic (T6-T12) segment of spinal cord were dissected and isolated, and total RNA was extracted using TRIzol reagent (Beijing Tiangen Biotech CO.). 6 $\mu \mathrm{g}$ total RNA was used as a template for cDNA synthesis and amplification using the First-Stand Synthesis System (BioPhotometer, Eppendorf, Hamburg, Germany) according to the manufacturer's instructions [35, 42-45]. The primers were designed with the Primer Express 3.0 software (Applied Biosystems), and the specific forward (F) and reverse (R) primer sequences were in Table 4. Experiments were evaluated in triplicate and repeated at least three times. The threshold cycle (CT) was used to estimate the amount of target mRNA. The comparative CT method with the formula for relative fold-change $=2^{-\Delta \Delta C T}$ was used to quantify the amplified transcripts.

\section{Data analysis}

Results are expressed as the mean \pm SEM. Statistical comparisons were performed with Mann-Whitney test. Statistical comparisons were performed using unpaired 
or paired Student's t tests or two-way repeated measures ANOVA. $\mathrm{P}<0.05, \mathrm{p}<0.01$, and $\mathrm{p}<0.001$ represent statistically significant differences.

\section{Abbreviations}

BDL: bile duct ligation; GO: gene ontology; KEGG: Kyoto Encyclopedia of Genes and Genomes; SD: Sprague-Dawley; RT-qPCR: quantitative real-time PCR; DEGs:differentially expressed genes; lncRNAs: long noncoding RNAs.

\section{ACKNOWLEDGMENTS}

This work was supported by grants from National Natural Science Foundation of P.R. China (No. 81670240, 81271766), National Natural Science Foundation of Hubei Province (No. 2016CFB625, 2016CFB324), Health and family planning commission in Hubei Province scientific research project (No.WJ2017M131 to M.C), Major project in Hebei Province education science (No.ZD 2016006 to S.L), and Special Fund of Fundamental Scientific Research Business Expense for Higher School of Central Government (2012 TS060 to H.X).

\section{CONFLICTS OF INTEREST}

The authors have no conflicts of interest related to this paper.

\section{REFERENCES}

1. Yuan YW, Wang L, Lu ZY, Long Y, Jiao YF, Xia Q, Wen DX, Yu WF. Overexcited MaxiK and KATP channels underlie obstructive jaundice-induced vasoconstrictor hyporeactivity of arterial smooth muscle. Sci Rep. 2016; 6:39246

2. Wang L, Yu WF. Obstructive jaundice and perioperative management. Acta Anaesthesiol Taiwan. 2014; 52:22-29.

3. Kremer AE, Martens JJ, Kulik W, Rueff F, Kuiper EM, van Buuren HR, van Erpecum KJ, Kondrackiene J, Prieto J, Rust C, Geenes VL, Williamson C, Moolenaar WH, et al. Lysophosphatidic acid is a potential mediator of cholestatic pruritus. Gastroenterology. 2010; 139:1008-1018, 1018 e1001.

4. Wang ZM, Zhang P, Lin MJ, Tan B, Qiu HB, Yu WF. Influence of obstructive jaundice on pharmacodynamics of rocuronium. PLoS One. 2013; 8:e78052.

5. Ren HM, Yang LQ, Liu ZQ, Chen CY, Cheung CW, Tao $\mathrm{KM}$, Song JG, Chen WR, Yu WF. In vivo and ex vivo effects of propofol on myocardial performance in rats with obstructive jaundice. BMC Gastroenterol. 2011; 11:144.

6. Tao KM, Tao Y, Chen CY, Yang LQ, Lu ZJ, Sun YM, Huang $\mathrm{SD}, \mathrm{Yu}$ WF. Proteinase-activated receptor 1 contributed to up-regulation of enkephalin in keratinocytes of patients with obstructive jaundice. Anesthesiology. 2014; 121:127-139.

7. Alemi F, Kwon E, Poole DP, Lieu T, Lyo V, Cattaruzza F, Cevikbas F, Steinhoff M, Nassini R, Materazzi S, GuerreroAlba R, Valdez-Morales E, Cottrell GS, et al. The TGR5 receptor mediates bile acid-induced itch and analgesia. J Clin Invest. 2013; 123:1513-1530.

8. Lieu T, Jayaweera G, Zhao P, Poole DP, Jensen D, Grace M, McIntyre P, Bron R, Wilson YM, Krappitz M, Haerteis S, Korbmacher C, Steinhoff MS, et al. The bile acid receptor TGR5 activates the TRPA1 channel to induce itch in mice. Gastroenterology. 2014; 147:1417-1428.

9. Dawson PA, Karpen SJ. Bile acids reach out to the spinal cord: new insights to the pathogenesis of itch and analgesia in cholestatic liver disease. Hepatology. 2014; 59:1638-1641.

10. Lieu T, Jayaweera G, Bunnett NW. GPBA: a GPCR for bile acids and an emerging therapeutic target for disorders of digestion and sensation. $\mathrm{Br} \mathrm{J}$ Pharmacol. 2014; 171:1156-1166.

11. He ZG, Liu BW, Li ZX, Liu C, Xiang HB. Altered expression profiling of spinal genes modulated by compound $48 / 80$ in a mouse itch model. J Anesth Perioper Med. 2017; 4:220-224.

12. Liu BW, Li ZX, He ZG, Liu C, Xiong J, Xiang HB. Altered expression of target genes of spinal cord in different itch models compared with capsaicin assessed by RT-qPCR validation. Oncotarget. 2017; 8:74423-74433. https://doi. org/10.18632/oncotarget.20148.

13. Liu QQ, Liu H, He ZG, Zhang SJ, Liu BW, Wang L, Qiu WH, Xu Q, Xiang HB, Lv YM. Differential gene and lncRNA expression in the lower thoracic spinal cord following ischemia/reperfusion-induced acute kidney injury in rats. Oncotarget. 2017; 8:53465-53481. https:// doi.org/10.18632/oncotarget.18584.

14. Li ZX, Liu BW, He ZG, Xiang HB. Melanocortin-4 receptor regulation of pain. Biochim Biophys Acta. 2017; 1863:2515-2522.

15. Liu T, He Z, Tian X, Kamal GM, Li Z, Liu Z, Liu H, Xu F, Wang J, Xiang H. Specific patterns of spinal metabolites underlying alpha-Me-5-HT-evoked pruritus compared with histamine and capsaicin assessed by proton nuclear magnetic resonance spectroscopy. Biochim Biophys Acta. 2017; 1863:1222-1230.

16. Liu TT, Liu BW, He ZG, Feng L, Liu SG, Xiang HB. Delineation of the central melanocortin circuitry controlling the kidneys by a virally mediated transsynaptic tracing study in transgenic mouse model. Oncotarget. 2016; 7:69256-69266. https://doi.org/10.18632/oncotarget.11956.

17. Zhao X, Tang Z, Zhang H, Atianjoh FE, Zhao JY, Liang L, Wang W, Guan X, Kao SC, Tiwari V, Gao YJ, Hoffman $\mathrm{PN}$, Cui $\mathrm{H}$, et al. A long noncoding RNA contributes to neuropathic pain by silencing Kcna2 in primary afferent neurons. Nat Neurosci. 2013; 16:1024-1031. 
18. Jiang BC, Sun WX, He LN, Cao DL, Zhang ZJ, Gao YJ. Identification of IncRNA expression profile in the spinal cord of mice following spinal nerve ligation-induced neuropathic pain. Mol Pain. 2015; 11:43.

19. Zhao B, Lu M, Wang D, Li H, He X. Genome-wide identification of long noncoding RNAs in human intervertebral disc degeneration by RNA sequencing. Biomed Res Int. 2016; 2016:3684875.

20. Wan ZY, Song F, Sun Z, Chen YF, Zhang WL, Samartzis D, Ma CJ, Che L, Liu X, Ali MA, Wang HQ, Luo ZJ. Aberrantly expressed long noncoding RNAs in human intervertebral disc degeneration: a microarray related study. Arthritis Res Ther. 2014; 16:465.

21. Lagarde J, Uszczynska-Ratajczak B, Santoyo-Lopez J, Gonzalez JM, Tapanari E, Mudge JM, Steward CA, Wilming L, Tanzer A, Howald C, Chrast J, Vela-Boza A, Rueda A, et al. Extension of human lncRNA transcripts by RACE coupled with long-read high-throughput sequencing (RACE-Seq). Nat Commun. 2016; 7:12339.

22. Denk F, Crow M, Didangelos A, Lopes DM, McMahon SB. Persistent alterations in microglial enhancers in a model of chronic pain. Cell Rep. 2016; 15:1771-1781.

23. Suarez-Farinas M, Ungar B, Correa da Rosa J, Ewald DA, Rozenblit M, Gonzalez J, Xu H, Zheng X, Peng X, Estrada YD, Dillon SR, Krueger JG, Guttman-Yassky E. RNA sequencing atopic dermatitis transcriptome profiling provides insights into novel disease mechanisms with potential therapeutic implications. J Allergy Clin Immunol. 2015; 135:1218-1227.

24. Ouyang P, Wang S, Zhang H, Huang Z, Wei P, Zhang Y, Wu Z, Li T. Microarray analysis of differentially expressed genes in L929 mouse fibroblast cells exposed to leptin and hypoxia. Mol Med Rep. 2017; 16:181-191.

25. Lee SS, Pak JM, Medlicott SM, Bomzon A. Vasodilatory responses of isolated arteries of cirrhotic rats. Clin Sci (Lond). 1995; 89:227-232.

26. Hasanein P, Parviz M, Keshavarz M, Javanmardi K, Allahtavakoli M, Ghaseminejad M. Modulation of cholestasis-induced antinociception in rats by two NMDA receptor antagonists: MK-801 and magnesium sulfate. Eur J Pharmacol. 2007; 554:123-127.

27. Rastegar H, Homayoun H, Afifi M, Rezayat M, Dehpour AR. Modulation of cholestasis-induced antinociception by CCK receptor agonists and antagonists. Eur Neuropsychopharmacol. 2002; 12:111-118.

28. Nelson L, Vergnolle N, D'Mello C, Chapman K, Le T, Swain MG. Endogenous opioid-mediated antinociception in cholestatic mice is peripherally, not centrally, mediated. J Hepatol. 2006; 44:1141-1149.

29. Vicuna L, Strochlic DE, Latremoliere A, Bali KK, Simonetti M, Husainie D, Prokosch S, Riva P, Griffin RS, Njoo C, Gehrig S, Mall MA, Arnold B, et al. The serine protease inhibitor SerpinA3N attenuates neuropathic pain by inhibiting T cell-derived leukocyte elastase. Nat Med. 2015; 21:518-523.

30. Belghiti M, Estevez-Herrera J, Gimenez-Garzo C, Gonzalez-Usano A, Montoliu C, Ferrer-Montiel A, Felipo V, Planells-Cases R. Potentiation of the transient receptor potential vanilloid 1 channel contributes to pruritogenesis in a rat model of liver disease. J Biol Chem. 2013; 288:9675-9685.

31. Tian B, Wang XL, Huang Y, Chen LH, Cheng RX, Zhou FM, Guo R, Li JC, Liu T. Peripheral and spinal 5-HT receptors participate in cholestatic itch and antinociception induced by bile duct ligation in rats. Sci Rep. 2016; 6:36286.

32. Lee $\mathrm{E}$. The effect of obstructive jaundice on the migration of reticulo-endothelial cells and fibroblasts into early experimental granulomata. Br J Surg. 1972; 59:875-877.

33. Karadeniz G, Acikgoz S, Tekin IO, Tascylar O, Gun BD, Comert M. Oxidized low-density-lipoprotein accumulation is associated with liver fibrosis in experimental cholestasis. Clinics (Sao Paulo). 2008; 63:531-540.

34. Nasehi M, Amin Yavari S, Zarrindast MR. Synergistic effects between CA1 mu opioid and dopamine D1-like receptors in impaired passive avoidance performance induced by hepatic encephalopathy in mice. Psychopharmacology (Berl). 2013; 227:553-566.

35. Guan XH, Fu QC, Shi D, Bu HL, Song ZP, Xiong BR, Shu B, Xiang HB, Xu B, Manyande A, Cao F, Tian YK. Activation of spinal chemokine receptor CXCR3 mediates bone cancer pain through an Akt-ERK crosstalk pathway in rats. Exp Neurol. 2015; 263:39-49.

36. Song Z, Xiong B, Zheng H, Manyande A, Guan X, Cao F, Ren L, Zhou Y, Ye D, Tian Y. STAT1 as a downstream mediator of ERK signaling contributes to bone cancer pain by regulating MHC II expression in spinal microglia. Brain Behav Immun. 2017; 60:161-173.

37. Dominguez CA, Lidman O, Hao JX, Diez M, Tuncel J, Olsson T, Wiesenfeld-Hallin Z, Piehl F, Xu XJ. Genetic analysis of neuropathic pain-like behavior following peripheral nerve injury suggests a role of the major histocompatibility complex in development of allodynia. Pain. 2008; 136:313-319.

38. Li Z, Yin P, Chen J, Li C, Liu J, Rambojan H, Luo F. Activation of the extracellular signal-regulated kinase in the amygdale modulates fentanyl-induced hypersensitivity in rats. J Pain. 2017; 18:188-199.

39. Zhu B, Xu M, Shi H, Gao X, Liang P. Genomewide identification of lncRNAs associated with chlorantraniliprole resistance in diamondback moth Plutella xylostella (L.). BMC Genomics. 2017; 18:380.

40. Li D, Ji Y, Wang F, Wang Y, Wang M, Zhang C, Zhang W, Lu Z, Sun C, Ahmed MF, He N, Jin K, Cheng S, et al. Regulation of crucial lncRNAs in differentiation of chicken embryonic stem cells to spermatogonia stem cells. Anim Genet. 2017; 48:191-204. 
41. Wang CY, Liu SR, Zhang XY, Ma YJ, Hu CG, Zhang JZ. Genome-wide screening and characterization of long non-coding RNAs involved in flowering development of trifoliate orange (Poncirus trifoliata L. Raf.). Sci Rep. 2017; 7:43226.

42. Ke C, Gao F, Tian X, Li C, Shi D, He W, Tian Y. Slit2/ Robo1 mediation of synaptic plasticity contributes to bone cancer pain. Mol Neurobiol. 2017; 54:295-307.

43. Bu H, Shu B, Gao F, Liu C, Guan X, Ke C, Cao F, Hinton AO Jr, Xiang H, Yang H, Tian X, Tian Y. Spinal IFNgamma-induced protein-10 (CXCL10) mediates metastatic breast cancer-induced bone pain by activation of microglia in rat models. Breast Cancer Res Treat. 2014; 143:255-263.
44. Xu B, Guan XH, Yu JX, Lv J, Zhang HX, Fu QC, Xiang HB, Bu HL, Shi D, Shu B, Qin LS, Manyande A, Tian YK. Activation of spinal phosphatidylinositol 3-kinase/protein kinase B mediates pain behavior induced by plantar incision in mice. Exp Neurol. 2014; 255:71-82.

45. Fu Q, Shi D, Zhou Y, Zheng H, Xiang H, Tian X, Gao F, Manyande A, Cao F, Tian Y, Ye D. MHC-I promotes apoptosis of GABAergic interneurons in the spinal dorsal horn and contributes to cancer induced bone pain. Exp Neurol. 2016; 286:12-20. 